\title{
Effect of corrugated wall combined with backward-facing step channel on fluid flow and heat transfer
}

\begin{abstract}
The turbulent fluid flow and heat transfer were numerically studied through backward-facing step combined with various corrugated walls. The governing equation was solved using Finite Volume Method (FVM) and the SIMPLE algorithm was applied to investigate the effect of backward-facing step with corrugated downstream on heat transfer characteristics. A constant heat flux was applied on the downstream wall, while the other walls were considered as adiabatic surfaces. Parameters such as corrugated design, amplitude height (1, 2, 3, 4 and $5 \mathrm{~mm}$ ) and Reynolds number (Re) in the range of 5000 to 20,000 were used. The performance evaluation criteria (PEC) were estimated to show the heat transfer augmentation. The results indicated that using a corrugated wall with a backward-facing step increased significantly the heat transfer accompanied by a slight increase in the skin friction coefficient simultaneously. The best heat transfer augmentation was observed for the trapezoidal corrugation at $4 \mathrm{~mm}$ amplitude height and $20 \mathrm{~mm}$ pitch diameter. Combining the corrugated wall with backward-facing step enhanced the Nusselt number $(\mathrm{Nu})$ up to $62 \%$ at $\mathrm{Re}=5000$. The performance evaluation criteria increased with the increase of amplitude height until it reached $4 \mathrm{~mm}$ and then decreased steeply.
\end{abstract}

Keyword: Backward-facing step; Corrugated wall; Fluid flow; Heat transfer enhancement 